\title{
Absence of ferromagnetism in Co and Mn substituted polycrystalline $\mathrm{ZnO}$
}

\author{
G. Lawe:* \\ Los Alamos National Laboratories, Los Alamos, New Mexico, 87545 \\ A.S. Risbud $\AA^{\dagger}$ \\ Materials Department \\ University of California, Santa Barbara, CA 93106 \\ A.P. Ramired \\ Bell Laboratories, Lucent Technologies, Murray Hill, NJ 07974 \\ Ram Seshadr \$ \\ Materials Department and Materials Research Laboratory \\ University of California, Santa Barbara, CA 93106
}

\begin{abstract}
We discuss the properties of semiconducting bulk $\mathrm{ZnO}$ when substituted with the magnetic transition metal ions $\mathrm{Mn}$ and Co, with substituent fraction ranging from $x=0.02$ to $x=0.15$. The magnetic properties were measured as a function of magnetic field and temperature and we find no evidence for magnetic ordering in these systems down to $T=2 \mathrm{~K}$. The magnetization can be fit by the sum of a Curie-Weiss term with a Weiss temperature of $\Theta \gg 100 \mathrm{~K}$ and a Curie term. We attribute this behavior to contributions from both $t \mathrm{M}$ ions with $t \mathrm{M}$ nearest neighbors and from isolated spins. This particular functional form for the susceptibility is used to explain why no ordering is observed in $t \mathrm{M}$ substituted $\mathrm{ZnO}$ samples despite the large values of the Weiss temperature. We also discuss in detail the methods we used to minimize any impurity contributions to the magnetic signal.

PACS numbers: 75.50.Pp, 75.60.-d
\end{abstract}

The ability to control spin as well as electric charge is a cornerstone of next generation spintronic devices. Attempts to inject spin-polarized current into non-magnetic semiconductors using metallic ferromagnetic contacts have met with mixed success $\frac{1.2}{.}$ Efficient spin injection between metallic and semiconducting systems appears to be possible only with an insulating tunnel junction separating the two $\frac{3.4 .5}{4}$ This complication could be removed by using a high temperature ferromagnetic semiconductor. Such a material would play a crucial role in the development of spintronic devices. Mn-substituted GaAs has been observed to have a ferromagnetic transition temperature of up to $172 \mathrm{~K}, \frac{6}{,}$ but reports of transition tempera-

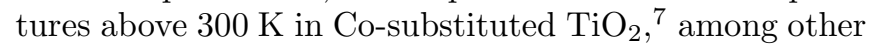
systems may be attributed to sample inhomogeneities $\underline{\underline{8}}$ Recent work on organic spin-valves ${ }^{9}$ have shown promising results, but in this report we concentrate on semiconducting metal oxides.

One material which shows particular promise for yielding a suitable ferromagnetic semiconductor is $\mathrm{ZnO}$. Zinc oxide is a wide bandgap $(3.3 \mathrm{eV})$ semiconductor, so a ferromagnetic version could also be used as a material for magneto-optical devices. ZnO substituted with $5 \% \mathrm{Mn}$ was predicted to order magnetically above $300 \mathrm{~K}, 10$ and the ground state for $\mathrm{ZnO}$ substituted with other transition metal $(t \mathrm{M})$ ions is predicted to be ferromagnetic ${ }^{11}$ Recently, there has been work in investigating the importance of $p$-type doping for producing ferromagnetic behavior in $\mathrm{ZnO} 12$ There have been a range of experiments done on Mn-substituted $\mathrm{ZnO}, \frac{13.14 .15}{2}$ with some investigation of $\mathrm{ZnO}$ substituted with other transition metals ${ }^{15.16 .17 .18}$ Several measurements on Co- and Mnsubstituted $\mathrm{ZnO}$ grown in thin films have found evidence for ferromagnetic behavior, but other measurements find no magnetic transitions. It is thought that clustering of the magnetic ions into an impurity phase might be responsible for these features $\frac{19}{\underline{n}}$ One recent report finds evidence for room temperature ferromagnetism in bulk $\mathrm{Mn}: \mathrm{ZnO}$ prepared using low temperature techniques ${ }^{20}$. Ferromagnetism has also been observed in Co:ZnO semiconducting quantum dots 21 , but the magnetic properties of nanoparticles can differ from bulk behavior ${ }^{22}$.

In light of these discrepancies in earlier measurements on the magnetic properties of $t \mathrm{M}$ substituted $\mathrm{ZnO}$, we have undertaken a study of bulk $\mathrm{Mn}_{x} \mathrm{Zn}_{1-x} \mathrm{O}$ and $\mathrm{Co}_{x} \mathrm{Zn}_{1-x} \mathrm{O}$ samples which we hope will resolve some of the ambiguities surrounding reports of ferromagnetism in these systems. We investigate bulk samples to minimize the contribution of extrinsic effects from surface impurities. Furthermore, by exploring samples with a range of $t \mathrm{M}$ fractions prepared using the same technique, we can investigate systematic trends in these compounds. Finally, our measurements are structured to search for and eliminate any possible contributions from the magnetic impurities which have plagued earlier studies. Specifically, investigating the differential high field susceptibility, as described below, eliminates any potential contributions to the signal from magnetic impurities not detected by our characterization techniques. This is necessary because magnetic measurements are a much more sensitive 


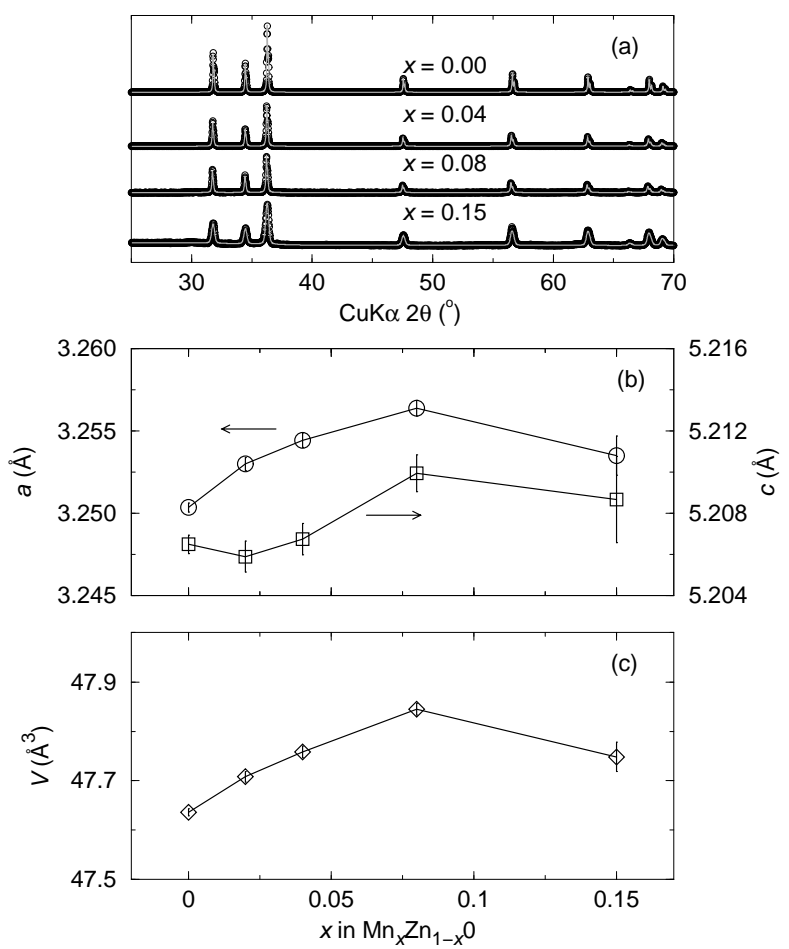

FIG. 1: (a) X-ray diffraction patterns (experimental data as circles and Rietveld simulations as lines) of $\mathrm{Mn}_{x} \mathrm{Zn}_{1-x} \mathrm{O}$ for different $x$ values as indicated in figure. (b) Evolution of the $a$ and $c$ cell parameters with increasing $x$ in $\mathrm{Mn}_{x} \mathrm{Zn}_{1-x} \mathrm{O}$. (c) Evolution of the cell volume with $x$. In (b) and (c) $3 \sigma$ error bars are indicated.

probe of ferromagnetic impurities than other characterization techniques.

In order to investigate the magnetic properties of $\mathrm{ZnO}: t \mathrm{M}$, we examined bulk samples of $\mathrm{Mn}_{x} \mathrm{Zn}_{1-x} \mathrm{O}$ and $\mathrm{Co}_{x} \mathrm{Zn}_{1-x} \mathrm{O}$ with $x$ ranging from 0.02 to 0.15 . It has been found previously that substitution above these levels leads to the development of a spinel phase related to $\mathrm{Co}_{3} \mathrm{O}_{4}$ in $\mathrm{Co}_{x} \mathrm{Zn}_{1-x} \mathrm{O}^{16}$ so we confine our experiments to smaller values of $x$. To obtain bulk $\mathrm{Mn}_{x} \mathrm{Zn}_{1-x} \mathrm{O}$ and $\mathrm{Co}_{x} \mathrm{Zn}_{1-x} \mathrm{O}$ samples, a single-source crystalline precursor is useful in order to ensure random atomic scale mixing of $\mathrm{Zn}^{2+}$ and $\mathrm{Mn}^{2+} / \mathrm{Co}^{2+}$ ions on lattice sites prior to decomposition. In addition, decomposition of oxalate precursors allows the removal of carbon as $\mathrm{CO}$ and $\mathrm{CO}_{2}$, leaving a phase-pure oxide product. Preparation and evidence for the formation of homogeneous solid solutions in bulk $\mathrm{Co}_{x} \mathrm{Zn}_{1-x} \mathrm{O}$ has been previously described in detail ${ }^{16} \mathrm{In}$ a similar manner, $\mathrm{Zn}_{1-x} \mathrm{Mn}_{x}\left(\mathrm{C}_{2} \mathrm{O}_{4}\right) \cdot 2 \mathrm{H}_{2} \mathrm{O}$ oxalate precursors were made with $x=0.02$ through $x=$ 0.15 . These precursors were decomposed in air at 1373 $\mathrm{K}$ for 15 minutes (with the sample placed and pulled out of the furnace at temperature). We performed elemental X-ray analysis on the Co substituted samples, confirming the Co fractions.

Powder X-ray diffraction patterns were recorded on a Scintag X2 diffractometer in the Bragg-Brentano config- uration using $\mathrm{CuK} \alpha$ radiation. Data were collected using a step scan of $0.015^{\circ}$ in $2 \theta$ and subject to Rietveld profile analysis using the XND Rietveld code ${ }^{23}$ Powder X-ray diffraction data are shown in Fig. [1 for the compositions $x=0.00,0.04,0.08$ and 0.15 for $\mathrm{Mn}_{x} \mathrm{Zn}_{1-x} \mathrm{O}$. Points are data and the solid lines are Rietveld fits. None of the samples in this compositional range showed any evidence for impurity phases. As the amount of Mn increases, the X-ray peak widths increase suggesting that crystalline correlation is decreased. This is consistent with the low natural solubility of $\mathrm{Mn}^{2+}$ in the $\mathrm{ZnO}$ lattice. Evolution of lattice parameters, as obtained from the Rietveld refinement, with $x$ is shown for the $\mathrm{Mn}_{x} \mathrm{Zn}_{1-x} \mathrm{O}$ in panel (b) and the evolution of the cell volume in panel (c). The cell parameter evolution is not simple; the $a$ cell parameter increases linearly with $x$ only from $x=0$ to $x=0.08$, which seems to be a limiting composition. The $c$ parameter first decreases slightly, and then increases. Again, $x=0.08$ seems to be a limiting composition. Substitution of the smaller $\mathrm{Zn}^{2+}$ ion (radius $=0.60 \AA$ ) by the larger $\mathrm{Mn}^{2+}$ ion (radius $=0.66 \AA$ ) should result in an increase in the unit cell volume. This increase is once again, systematic till $x=0.08$ as seen in Fig. 1 Slight differences are seen in our results from those recently published by Kolesnik, Dabrowski, and Mais, 18 who report, for $x=0,0.05,0.10,0.15$ and 0.20 , that $a, c$ and $V$ increase smoothly with $x$ but the $x=0.15$ sample is not single-phase. Because of this, we restrict our magnetic measurements on $\mathrm{Mn}_{x} \mathrm{Zn}_{1-x} \mathrm{O}$, to samples with $x$ below 0.10 .

Magnetic measurements were performed using a commercial Superconducting Quantum Interference Device (SQUID) magnetometer (Quantum Design MPMS). For each measurement, we used roughly $30 \mathrm{mg}$ of sample which gave a signal over three orders of magnitude larger than the magnetic background of the sample holder. We measured both the magnetization as a function of field at fixed temperature, and the temperature dependence of the susceptibility. In order to accurately determine the intrinsic magnetization of the sample as a function of temperature, we measured the differential susceptibility by subtracting the magnetic moment at $B=1 \mathrm{~T}$ from the moment measured at $B=2 \mathrm{~T}$ at each temperature. The background susceptibility of $\mathrm{ZnO}$ was measured separately and subtracted. This background contribution was roughly $10 \%$ of the total susceptibility at high temperatures.

Because impurity contributions have previously been misidentified as intrinsic effects $\frac{19}{19}$, it is vital to ensure that we are unable to detect any impurities in the sample, and also that any impurity contribution to the magnetization is removed. We know from XRD that any crystalline impurity fraction must be below the $1 \%$ level. In addition, low field susceptibility measurements as function of magnetic field at fixed temperature show no deviation from strictly linear behavior. We take this as evidence for both the lack of impurities, and that the $t \mathrm{M}$ ions are fully substituting into the lattice. As a fi- 


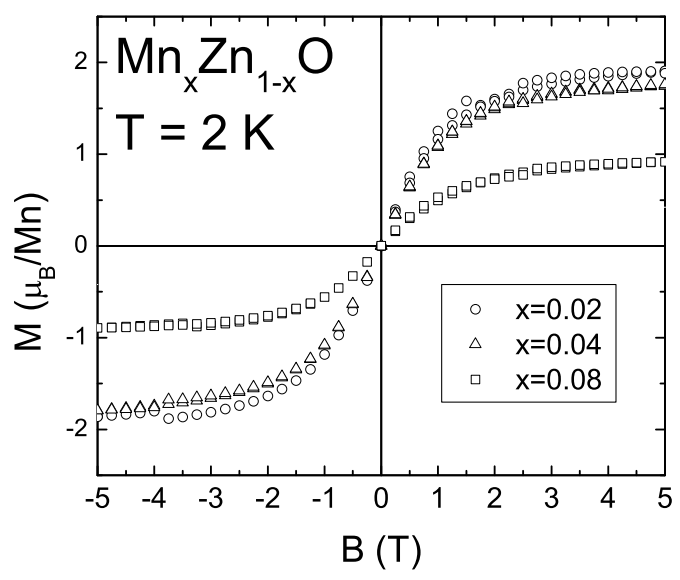

FIG. 2: Magnetic moment per $\mathrm{Mn}^{2+}$ as a function of external field at $T=2 \mathrm{~K}$. The magnetization of $\mathrm{Mn}_{x} \mathrm{Zn}_{1-x} \mathrm{O}$ is plotted for four values of $x$ ranging from $x=0.02$ to $x=0.08$.

nal check for impurities, we measured specific heat of the $x=0.15$ sample (which would be expected to have the largest impurity contributions), since this is a bulk probe of magnetic order, even down to very small impurity fractions. The absence of any signal in specific heat arising from long range order in magnetic impurities is consistent with the magnetic measurements and suggests that we are probing the intrinsic properties of $t \mathrm{M}$-substituted $\mathrm{ZnO}$. Additionally, by measuring the differential susceptibility as described above, we can be certain that any spurious contribution to the signal from ferromagnetic impurity clusters not identified by the characterizations discussed above will be eliminated.

We plot the magnetization as a function of magnetic field at $T=2 \mathrm{~K}$ for $\mathrm{Mn}_{x} \mathrm{Zn}_{1-x} \mathrm{O}$ for different values of $x$ in figure 2 These magnetization data suggest that there are contributions to the magnetic signal from both free spins and spins associated with antiferromagnetic clusters. The suppression of the net magnetic moment seen as $x$ is increased is opposite to expectations based on free $\mathrm{Mn}$ impurity spins. Since $x=0.08$ is within a factor of two of the $3 \mathrm{D}$ percolation threshold on the $\mathrm{ZnO}$ lattice, one would expect to see effects from clustering. This would lead to a reduction of the measured moment if the pure $\mathrm{Mn}$ end-member were antiferromagnetic. We observe that the moment is large for the lowest concentrations $(x=0.02$ and $x=0.04)$, but becomes smaller as the substituent concentration increases. The moment plotted in Figure 2 arises predominantly from free spins in the system. With increasing $x$, the fraction of Mn ions belonging to antiferromagnetic clusters which do not contribute to the magnetic signal increases, which reduces the net magnetization. This behavior is very similar to that previously observed in $\mathrm{Co}_{x} \mathrm{Zn}_{1-x} \mathrm{O} \stackrel{16}{\rightleftharpoons}$. Furthermore, the $M(B)$ curves shown in figure 2 show no evidence for

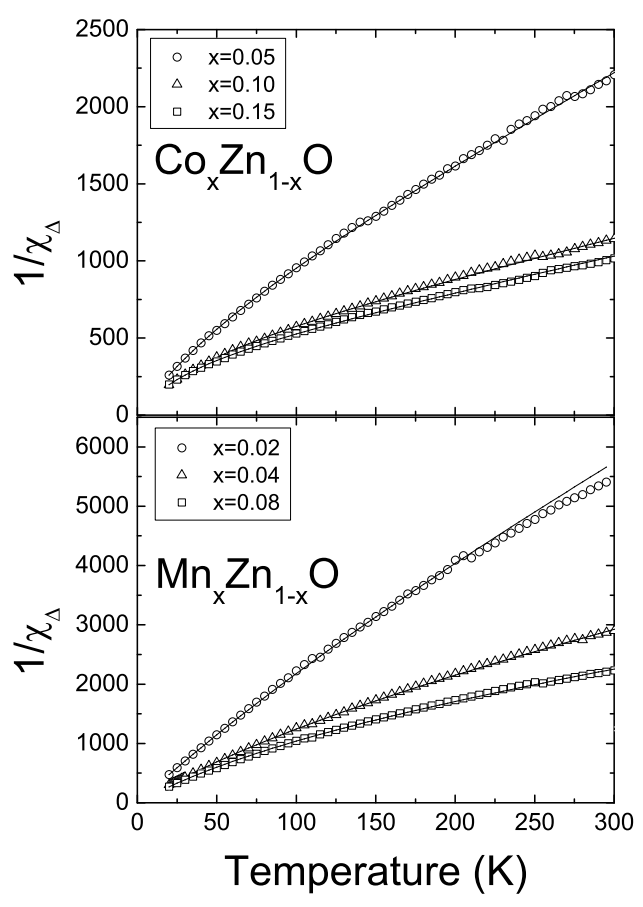

FIG. 3: Inverse susceptibility for $\mathrm{Co}_{x} \mathrm{Zn}_{1-x} \mathrm{O}$ (upper panel) and $\mathrm{Mn}_{x} \mathrm{Zn}_{1-x} \mathrm{O}$ (lower panel) as a function of temperature. The susceptibility was determined by looking at the difference in magnetization between $B=2 \mathrm{~T}$ and $B=1 \mathrm{~T}$. The solid lines show fits to the function defined in Eq. 1

magnetic hysteresis even at $T=2 \mathrm{~K}$; there is no ferromagnetic transition in $\mathrm{Mn}_{x} \mathrm{Zn}_{1-x} \mathrm{O}$ above this temperature. This observation is in contrast to a report on room temperature ferromagnetism in $\mathrm{Mn}_{x} \mathrm{Zn}_{1-x} \mathrm{O}$ at $x$ $=0.02,20$ which found evidence for a very small ferromagnetic moment in samples prepared under relatively low temperature conditions. The origin for this discrepancy is unclear, although there are suggestions that these earlier measurements were sensitive to unreacted manganese oxides $^{24}$

The temperature dependence of the magnetizations of $\mathrm{Mn}_{x} \mathrm{Zn}_{1-x} \mathrm{O}$ and $\mathrm{Co}_{x} \mathrm{Zn}_{1-x} \mathrm{O}$ are plotted in figure [3] as inverse susceptibility versus temperature. These plots show characteristic behavior observed in other $t \mathrm{M}$ substituted $\mathrm{ZnO}$ samples, namely a high temperature regime that appears to be close to linear, followed by significant curvature at lower temperatures. Furthermore, there is a systematic variation in the high temperature magnetization with substitution fraction. The samples with the smallest values of $x$ show larger values of $1 / \chi$, and the inverse susceptibility decreases monotonically with increasing $x$ as has been observed previously.

In order to analyze such data, it is typical to make a linear fit of the high temperature inverse susceptibility which models Curie-Weiss behavior. This approach is 
motivated by prior work on dilute magnetic semiconductors which predicts that the high temperature magnetization should follow a modified Curie-Weiss law, where the Curie constant and Curie-Weiss temperature are scaled by the substituent concentration $x{ }^{25}$ While this model appears to accurately predict the properties of Se and Te based semiconductors even at lower temperatures where there are significant deviations from Curie-Weiss behavior ${ }^{25.26}$, we find discrepancies when attempting to apply this formalism to $\mathrm{Co}_{x} \mathrm{Zn}_{1-x} \mathrm{O}$ and $\mathrm{Mn}_{x} \mathrm{Zn}_{1-x} \mathrm{O}$, particularly in fitting the low temperature susceptibility. Furthermore, applying this analysis to $\mathrm{ZnO}: t \mathrm{M}$ samples gives a wide range of very large values for the Weiss temperature (from $\Theta_{0}=960 \mathrm{~K}$ to $\Theta_{0}=1900 \mathrm{~K}$ for $\mathrm{Mn}_{x} \mathrm{Zn}_{1-x} \mathrm{O}^{13}$ ) despite the absence of any magnetic order above $T=20 \mathrm{~K}$.

To interpret our results, we propose a heuristic model based on two sets of substituent $t \mathrm{M}$ spins, motivated by Figure 2 and the subsequent discussion. There is perhaps a hint of such behavior observed in NMR studies on $\mathrm{Cd}_{1-x} \mathrm{Co}_{x} \mathrm{~S}$ and $\mathrm{Cd}_{1-x} \mathrm{Fe}_{x} \mathrm{~S}$ where it was observed that the splitting of the ${ }^{113} \mathrm{Cd}$ spectra can be explained by appealing to different relative connections to the paramagnetic substituents ${ }^{27}$ We assume that one of these subsets of spins (those $t \mathrm{M}$ ions with no $t \mathrm{M}$ nearest neighbors) is completely free, so the susceptibility follows a simple Curie behavior. The second set of spins (those $t \mathrm{M}$ ions with at least one $t \mathrm{M}$ nearest neighbor) is however affected by mean field interactions with a susceptibility which is expressed as a Curie-Weiss function. We find that separating the $t \mathrm{M}$ ions into two non-interacting subsets, namely free spins and clusters, gives an excellent fit to the susceptibility over the entire temperature range. We also allow for the possibility that the Curie constants will be different for the two terms. In particular, we assume

$$
\chi=\frac{C_{1}}{T}+\frac{C_{2}}{T+\Theta}
$$

in order to fit the magnetization data for $\mathrm{Co}_{x} \mathrm{Zn}_{1-x} \mathrm{O}$ and $\mathrm{Mn}_{x} \mathrm{Zn}_{1-x} \mathrm{O}$. The solid lines in figure 3 show the fits to Eq. 11 which accurately capture both the high temperature behavior and the increasing curvature in $\chi_{\Delta}^{-1}$ at lower temperatures.

Despite these fits requiring three adjustable parameters, this model provides insight into the magnetic properties of $\mathrm{Co}_{x} \mathrm{Zn}_{1-x} \mathrm{O}$ and $\mathrm{Mn}_{x} \mathrm{Zn}_{1-x} \mathrm{O}$. An important observation is that only a subset of the spins are affected by magnetic interactions. For Mn-substituted $\mathrm{ZnO}$, the value of $\Theta$ lies between roughly $+190 \mathrm{~K}$ and $+360 \mathrm{~K}$ for all samples measured. This suggests that the spins belonging to $t \mathrm{M}$ clusters are affected by antiferromagnetic mean field interactions, consistent with the $M(B)$ behavior plotted in Figure 2 Very similar properties are observed for $\mathrm{Co}_{x} \mathrm{Zn}_{1-x} \mathrm{O}$, but the value of $\Theta$ ranges from $+160 \mathrm{~K}(x=0.05)$ to $+250 \mathrm{~K}(x=0.15)$.

We plot the Curie constants $\left(C_{1}\right.$ and $\left.C_{2}\right)$ from these fits as a function of substituent concentration $x$ in figure

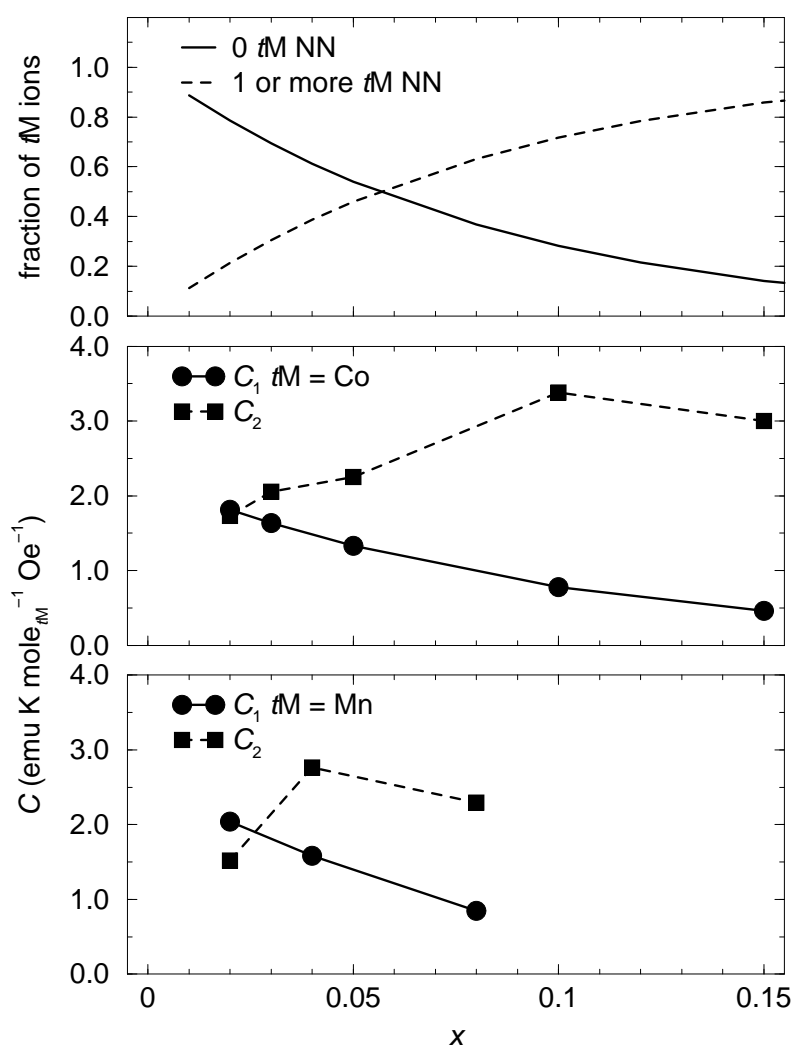

FIG. 4: Values of the Curie constants for $\mathrm{Co}_{x} \mathrm{Zn}_{1-x} \mathrm{O}$ (middle panel) and $\mathrm{Mn}_{x} \mathrm{Zn}_{1-x} \mathrm{O}$ (lower panel) extracted from the fits to Eq. 11 The solid symbols give the Curie constant corresponding to the interacting term in Eq. 11 while the open symbols correspond to the non-interacting term. Upper Panel: Computed distribution of $t \mathrm{M}$ ions on the cations sites of the wurtzite $\mathrm{ZnO}$ lattice with zero $t \mathrm{M}$ ion near-neighbors (solid line) and with one or more $t \mathrm{M}$ ion near-neighbors (dashed line), as a function of $x$. We assume random substitution of $t \mathrm{M}$ on the $\mathrm{Zn}$ site.

[4 which are plotted scaled to moles Co or Mn. There is a systematic increase of the contribution from the interacting term with increasing $x$, while the pure Curie term shows a corresponding decrease as the substituent concentration is raised. Since the explicit $x$ dependence of $C_{1}$ and $C_{2}$ has been taken into account by expressing these parameters in moles of Co or $\mathrm{Mn}$, this implies that the contribution of the interacting $\mathrm{Mn}$ or Co ions increases with the fraction of $t \mathrm{M}$ ions belonging to magnetic clusters. Conversely, the relative importance of the non-interacting contribution from Eq. [1 is reduced as the proportion of isolated spins decreases with increased substitution. This observation justifies our initial assumption that we can divide the magnetic substituents into two distinct subsets. However, it should be noted that the Curie constants we obtain with this analysis are rather different than what one would expect for free $\mathrm{Mn}^{2+}$ or $\mathrm{Co}^{2+}$ spins (4.38 and $1.87 \mathrm{emu} \mathrm{K} /$ mole resp.). At present we don't understand the origin of this dis- 
crepancy, although the degree of orbital quenching could be a relevant parameter. The top panel of the figure shows the fraction of $t \mathrm{M}$ ions with no $t \mathrm{M}$ ion nearest neighbors $(\mathrm{NN})$, and the fraction with at least one $t \mathrm{M}$ $\mathrm{NN}$, computed from a model with the $t \mathrm{M}$ ions randomly distributed on the $\mathrm{Zn}$ sites.

This analysis of the magnetic properties of $t \mathrm{M}$ substituted $\mathrm{ZnO}$ reveals several features. By investigating a series of compounds with different $t \mathrm{M}$ fractions, we find clear evidence for a systematic clustering of spins as the proportion of magnetic ions is increased. Additionally, we find that the dominant spin-spin interactions are antiferromagnetic, in agreement with other published results 13 . Finally, we offer a simple explanation as to why these strong antiferromagnetic interactions do not lead to long range spin ordering. If we assume that the spins are localized and have no itinerant character, the lack of magnetic order arises from geometrical considerations. The 3D site percolation threshold for an FCC lattice (having the same numbers of nearest neighbors as the wurtzite lattice) is $19.5 \%{ }^{28}$, which is significantly higher than the maximum $t \mathrm{M}$ substitution of $10 \%$ for these samples. Since the concentration of magnetic ions is well below the percolation threshold, we would not expect to find magnetic order in these systems, at least at temperatures commensurate with the interaction strengths (few hundred K).

In summary, our measurements on a series of $\mathrm{Co}_{x} \mathrm{Zn}_{1-x} \mathrm{O}$ and $\mathrm{Mn}_{x} \mathrm{Zn}_{1-x} \mathrm{O}$ samples with $x$ ranging from 0.01 to 0.15 show no evidence for a ferromagnetic transition in these systems above $T=2 \mathrm{~K}$. At low temperatures, the characteristic dependence of the magnetic moment in $\mathrm{Mn}_{x} \mathrm{Zn}_{1-x} \mathrm{O}$ on $B$ suggests that the dominant interactions are antiferromagnetic, as observed previously in $\mathrm{Co}_{x} \mathrm{Zn}_{1-x} \mathrm{O}$ samples prepared under similar conditions $\frac{16}{16}$ The important finding is that susceptibility data in both systems are fitted very well over a large temperature range by the sum of two Curie-Weiss functions. The fits yield a high temperature antiferromagnetic interaction $(\Theta \gg 100 \mathrm{~K})$ which we associate with NN interactions among the magnetic clusters, while the susceptibility of the spins with no magnetic nearest neighbors can be fitted very well by a simple Curie function. We hope to verify the presence of two distinct spin populations in $t \mathrm{M}$ substituted $\mathrm{ZnO}$ using techniques such as EPR or Mossbauer spectroscopy.

Experimentally, we find that the dominant interactions in these systems are antiferromagnetic consistent with other recent measurements on these compounds ${ }^{13.16}$ It is becoming increasingly difficult to envision a scenario whereby substitution of $\mathrm{ZnO}$ with either $\mathrm{Mn}$ or Co would produce a ferromagnetic ground state, at least at temperatures above a few $\mathrm{K}$. This is consistent with recent work highlighting the importance of hole substitution in $\mathrm{Co}_{x} \mathrm{Zn}_{1-x} \mathrm{O}$ or $\mathrm{Mn}_{x} \mathrm{Zn}_{1-x} \mathrm{O}$ to obtain carrier-mediated ferromagnetism. 12

\section{Acknowledgments}

A. S. R. is supported by the National Science Foundation IGERT program under the award DGE-9987618. We gratefully acknowledge support for the UCSB/LANL collaboration from the CARE program of the University of California. The work was also partially supported by the MRL program of the National Science Foundation under the Award No. DMR00-80034 and by the LDRD program at Los Alamos National Laboratory. Tsuyoshi Kimura and Nicola Spaldin are thanked for suggestions, discussions and encouragement.

* Electronic address: glawes@lanl.gov

† Electronic address: aditi@engineering.ucsb.edu

¥ Electronic address: apr@lucent.com

$\S$ Electronic address: seshadri@mrl.ucsb.edu

1 P.R. Hammar, B.R. Bennett, M.J. Yang, and M. Johnson, Phys. Rev. Lett. 83, 203 (1999).

2 F.G. Monzon, H.X. Tang, and M. L. Roukes, Phys. Rev. Lett. 84, 5022 (2000).

${ }^{3}$ D.L. Smith and R.N. Silver, Phys. Rev. B 64, 045323 (2001).

4 E.I. Rashba, Phys. Rev. B 62, R16267 (2000).

${ }^{5}$ V.F. Motsnyi, J. De Boeck, J. Das, W. Van Roy, G. Borghs, E. Goovaerts, and V.I. Safarov, Appl. Phys. Lett. 81, 265 (2002).

6 A.M. Nazmul, S. Sugahara, and M. Tanaka, Phys. Rev. B 67, 241308 (2003).

7 Y. Matsumoto, et al., Science 291, 854 (2001).

8 S.A. Chambers, T. Droubay, C.M. Wang, A.S. Lea, R.F.C. Farrow, L. Folks, V. Deline, and S. Anders Appl. Phys. Lett. 82, 1257 (2003).

9 Z.H. Xiong, Di Wu, Z. Valy Vardeny, and Jing Shi, Nature

427, 821 (2004)

10 T. Dietl, H. Ohno, F. Matsukura, J. Cibert, and D. Ferrand, Science 287, 1019 (2000).

11 K. Sato and H. Katayama-Yoshida, Jpn. J. Appl. Phys. 39, L555 (2000).

12 N.A. Spaldin, Phys. Rev. B. 69, 125201 (2004).

13 S. Kolesnik, B. Dabrowski, and J. Mais, J. Supercond. 15, 251 (2002).

14 T. Fukumura, Z. Jin, M. Kawasaki, T. Shono, T. Hasegawa, S. Koshihara, and H. Koinuma, Appl. Phys. Lett. 78, 958 (2001).

15 S.W. Yoon, S.-B. Cho, S.C. We, S. Yoon, B.J. Suh, H.K. Song, and Y.J. Shin, J. Appl. Phys. 93, 7879 (2002).

16 A.S. Risbud, N.A. Spaldin, Z.Q. Chen, S. Stemmer, and R. Seshadri, Phys. Rev. B 68, 205202 (2003).

17 W.H. Brumage, C.F. Dorman, and C.R. Quade, Phys. Rev. B 63, 104411 (2001).

18 S. Kolesnik, B. Dabrowksi, and J. Mais, J. Appl. Phys. 95, 2582 (2003).

19 J.H. Kim, H. Kim, D. Kim, Y.E. Ihm, and W.K. Choo, J. Appl. Phys. 92, 6066 (2002). 
20 P. Sharma, A. Gupta, F.J. Owens, K.V. Rao, R. Sharma, R. Ahuja, J.M. Osorio Guillen, B. Johannson, and G.A. Gehring, Nature Mater. 2, 673 (2003).

21 D.A. Schwartz, N.S. Norberg, Q.P. Nguyen, J.M. Parker, and D.R. Gamelin, J. Am. Chem. Soc. 125, 13205 (2003).

${ }^{22}$ G.H. Lee, S.H. Huh, J.W. Jeong, B.J. Choi, S.H. Kim, and H.-C. Ri, J. Am. Chem. Soc. 124, 12094 (2002).

23 J.-F. Bérar, Computer code XND, Freely available from http://www.ccp14.ac.uk

24 S. Kolesnik and B. Dabrowski, arXiv:cond-mat/0404186 v1
25 J. Spałek, A. Lewicki, Z. Tarnawaksi, J.K. Furdyna, R.R. Galazka, and Z. Obuszko, Phys. Rev. B 33, 3407 (1986).

26 J. Furdyna, N. Samarth, R.B. Frankel, and J. Spalek, Phys. Rev. B37, 3707 (1988).

27 V. Ladizhanski, V. Lyahovitskaya, and S. Vega, Phys. Rev. B 60, 8097 (1999).

28 R.B. Stinchcombe, Phase Transitions v.7, Academic Press: London, p. 151 (1983). 